\title{
Crystalline Order On Riemannian Manifolds With Variable Gaussian Curvature And Boundary
}

\author{
Luca Giomi* and Mark Bowick ${ }^{\dagger}$ \\ Department of Physics, Syracuse University, Syracuse, New York 13240-1130, USA
}

\begin{abstract}
We investigate the zero temperature structure of a crystalline monolayer constrained to lie on a two-dimensional Riemannian manifold with variable Gaussian curvature and boundary. A full analytical treatment is presented for the case of a paraboloid of revolution. Using the geometrical theory of topological defects in a continuum elastic background we find that the presence of a variable Gaussian curvature, combined with the additional constraint of a boundary, gives rise to a rich variety of phenomena beyond that known for spherical crystals. We also provide a numerical analysis of a system of classical particles interacting via a Coulomb potential on the surface of a paraboloid.
\end{abstract}

\section{INTRODUCTION}

Crystalline structures are ubiquitous in nature because ordered close-packed configurations frequently minimize the interaction energy between the component units of the system. In soft condensed matter systems, where curved or fluctuating geometries are energetically accessible, the interplay between order and geometry has been the subject of much attention in the last two decades [1$9]$. The existence of some preferred geometry, as for instance that arising from the growth of a crystalline monolayer on a rigid substrate, may influence the nature of the allowed order and, on the other hand, the formation of particular ordered structure may lead to a deformation in the geometry of the substrate (i.e. shape transition $[10,11])$. Self assembled systems such as colloidosomes [4] or thin films of block copolymers [12] are realizations of such non-Euclidean "soft" crystals. Protein subunits, which comprise the shells of spherical viruses, also provide an example in which the mechanical properties of the systems are affected by the formation of crystalline aggregates on surfaces equipped with a non-zero Gaussian curvature.

Some progress in understanding crystalline arrangements of particles interacting on a curved surface was achieved by the authors of $[2,13]$ in the context of the geometric theory of topological defects. The original interacting particle problem is mapped to a system of interacting disclination defects in a continuum elastic curved background. The defect-defect interaction is universal with the particle microscopic potential determining two free parameters, the Young modulus of the elastic background and the core energy of an elementary disclination [14].

In flat two-dimensional space particles almost always pack in triangular lattices, unless the interaction potential is carefully tuned to select some other lattice topology. The addition of non-zero Gaussian curvature to the

\footnotetext{
*Electronic address: lgiomi@physics.syr.edu

${ }^{\dagger}$ Electronic address: bowick@physics.syr.edu
}

problem introduces frustration in the sense that perfect planar crystalline order is now incompatible with the curvature of the surface. The most clear-cut example of such geometrical frustration is the case of spherical crystals $[3,8]$. The geometrical frustration in terms of lattice topology is revealed by the Euler theorem on the sphere which relates the number of vertices $(V)$, faces $(F)$ and edges $(E)$ of any convex polyhedron : $V-E+F=\chi$, with $\chi=2$ in the case of the 2 -sphere. This classical result of topology can be rephrased in a form that is particularly useful to describe the presence of defects in the lowest energy configuration of a curved crystal by defining a topological charge as the departure from the ideal coordination number of a planar triangular lattice: $q_{i}=6-c_{i}$, with $c_{i}$ the coordination number of the $i-$ th vertex. The Euler theorem states that the total disclination charge of any spherical lattice must be equal to six times the Euler characteristic $\chi$ :

$$
Q=\sum_{i=1}^{V} q_{i}=6 \chi=12
$$

Although identical particles with hard core repulsion in a plane typically pack into a triangular lattice with 6 -fold coordination, any triangulation of the sphere is required to have twelve 5-fold disclinations (provided we restrict ourselves to the energetically-preferred charge $q= \pm 1$ disclinations) as well as an arbitrary number of 6 -fold vertices. These twelve extra disclinations are the consequence of the geometrical frustration associated the the curvature of the sphere.

Topological defects can appear in spherical lattices in the form of isolated 5-fold disclinations placed at the vertices of an icosahedron, as frequently happens in the case of small viral capsids, or grouped into one-dimensional arrays of dislocations (tightly-bound $(5,7)$-fold disclination pairs). These arrays, also known as grain boundary "scars", appear on the sphere when the ratio $R / a$ ( $R$ radius of the sphere, a Euclidean lattice spacing) exceeds a particular threshold value approximately equal to 5 .

In this article we present an analytical and numerical study of the defect structure of a two-dimensional crystal 
constrained to lie on the surface of a paraboloid of revolution. The parabolic geometry introduces two novel and important features compared to the sphere: 1) a variable Gaussian curvature and 2) the presence of a boundary. Both these features must be treated properly for a thorough theoretical understanding.

The paper is organized as follows. In Sec. II we briefly review the geometrical approach of $[2,3,8]$ in which the basic degrees of freedom are the defects themselves rather than the interacting particles and we derive the zero-temperature energy of a paraboloidal crystal. This formalism has the advantage of reducing the number of degrees of freedom as well as being rather universal in the sense that it applies to a broad class of interacting potentials. In Sec. III we discuss the results obtained by the numerical minimization of the potential energy of a system of classical charged particles interacting via a Coulomb potential on the surface of a paraboloid in the light of the geometrical approach. Sec. IV will be devoted to conclusions.

\section{THE GEOMETRICAL APPROACH}

\section{A. The Elastic Free Energy}

Let $\mathbb{P}$ be the two-dimensional paraboloid of revolution in $\mathbb{R}^{3}$ described in parametric form by:

$$
\left\{\begin{array}{l}
x=r \cos \phi \\
y=r \sin \phi \\
z=\frac{h}{R^{2}} r^{2}
\end{array}\right.
$$

where $h$ is the height of the paraboloid and $R$ the maximum radius. In the following we will call $\kappa=2 h / R^{2}$ the normal curvature of the paraboloid at the origin. The metric tensor $g_{i j}$ (with determinant $g$ ) and the Gaussian curvature $K$ are given respectively by:

$$
\begin{gathered}
g_{i j}=\left(\begin{array}{cc}
1+\kappa^{2} r^{2} & 0 \\
0 & r^{2}
\end{array}\right) \\
K(r)=\frac{\kappa^{2}}{\left(1+\kappa^{2} r^{2}\right)^{2}} .
\end{gathered}
$$

Such paraboloidal surfaces serve as a good testing ground for exploring the effects of both variable Gaussian curvature and the presence of a boundary on the nature of crystalline order. From the topological point of view a paraboloid of revolution is equivalent to a disk. The Euler characteristic is thus $\chi=1$. The total topological charge, taking into account the preferred coordination on the boundary and in the interior, is given in this case by:

$$
Q=\sum_{i=1}^{N_{b}}\left(4-c_{i}\right)+\sum_{i=1}^{N_{i}}\left(6-c_{i}\right)=6
$$

where $N_{b}$ is the total number of disclinations on the boundary and $N_{i}$ is the number of disclinations in the interior of the paraboloid (in the following we will reserve the letter $N$ for the total number of defects and $V$ for the total number of vertices of the lattice). The elastic free energy of the crystal may be expressed in the form $[2,3,8,13]$ :

$$
F=F_{e l}+F_{c}+F_{0},
$$

where $F_{0}$ is the free energy of the defect-free monolayer and $F_{c}$ is the contribution to the free energy due to the core energy of disclinations and is proportional to the total number of defects. The elastic energy $F_{e l}$ associated with the defect interaction can be expressed in the form:

$$
F_{e l}=\frac{1}{2} Y \int d^{2} x d^{2} y G_{2 L}(\boldsymbol{x}, \boldsymbol{y}) \rho(\boldsymbol{x}) \rho(\boldsymbol{y}),
$$

where $Y$ is the Young modulus for the planar crystal. The quantity $\rho(\boldsymbol{x})$ has the meaning of the effective topological charge density:

$$
\rho(\boldsymbol{x})=\frac{\pi}{3} \sum_{i=1}^{N} q_{i} \delta\left(\boldsymbol{x}, \boldsymbol{x}_{i}\right)-K(\boldsymbol{x}),
$$

where $\delta\left(\boldsymbol{x}, \boldsymbol{x}_{i}\right)$ is Dirac delta function on the manifold: $\delta\left(\boldsymbol{x}, \boldsymbol{x}_{i}\right)=g^{-1 / 2} \prod_{i} \delta\left(\boldsymbol{x}-\boldsymbol{x}_{i}\right)$ and $G_{2 L}(\boldsymbol{x}, \boldsymbol{y})$ represents the Green's function for the covariant biharmonic operator on $\mathbb{P}$.

The calculation of the effective free energy (5) can be simplified if free boundary conditions are chosen. An application of the second Green identity to Eq.(6) leads straightforwardly to the form:

$$
F_{e l}=\frac{1}{2 Y} \int d^{2} x[\Delta \chi(\boldsymbol{x})]^{2},
$$

where $\chi(\boldsymbol{x})$ is the solution of the inhomogeneous biharmonic equation:

$$
\Delta^{2} \chi(\boldsymbol{x})=Y \rho(\boldsymbol{x}) \quad \boldsymbol{x} \in \mathbb{P}
$$

with boundary conditions

$$
\begin{aligned}
\chi(\boldsymbol{x}) & =0 & & \boldsymbol{x} \in \partial \mathbb{P} \\
\nu_{i} \nabla^{i} \chi(\boldsymbol{x}) & =0 & & \boldsymbol{x} \in \partial \mathbb{P},
\end{aligned}
$$

in which $\nabla^{i}$ is the usual contravariant derivative in the metric $g_{i j}$ and $\nu_{i}$ is the $i$-th component of the tangent vector $\boldsymbol{\nu}$ perpendicular to boundary. If the parametrization (2) is chosen, the normal vector $\boldsymbol{\nu}$ is simply given by $\boldsymbol{g}_{r} /\left|\boldsymbol{g}_{r}\right|$, with $\boldsymbol{g}_{r}=\partial_{r} \boldsymbol{x}$ the base vector associated with radial coordinate $r$. The solution of Eq.(9) will then be:

$$
\chi(\boldsymbol{x})=\int d^{2} y G_{L}(\boldsymbol{x}, \boldsymbol{y}) \Gamma(\boldsymbol{y}),
$$

where $G_{L}(\boldsymbol{x}, \boldsymbol{y})$ is the Green's function of the covariant Laplace operator on $\mathbb{P}$ with Dirichlet boundary conditions

$$
\begin{cases}\Delta G_{L}(\boldsymbol{x}, \cdot)=\delta(\boldsymbol{x}, \cdot) & \boldsymbol{x} \in \mathbb{P} \\ G_{L}(\boldsymbol{x}, \cdot)=0 & \boldsymbol{x} \in \partial \mathbb{P}\end{cases}
$$




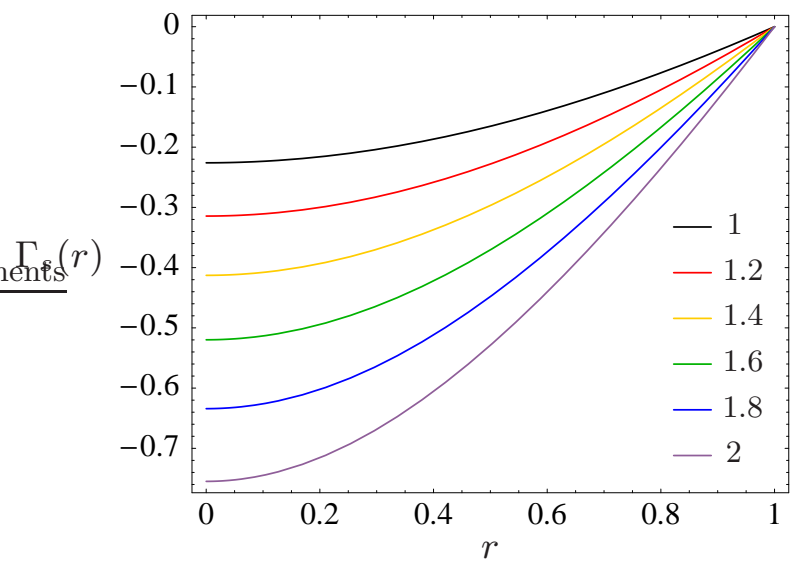

FIG. 1: The function $\Gamma_{s}(r)$ for different values of $\kappa$ in the range $1-2$.

and $\Gamma(\boldsymbol{x})=\Delta \chi(\boldsymbol{x})$ is the solution of the Poisson problem:

$$
\Delta \Gamma(\boldsymbol{x})=Y \rho(\boldsymbol{x})
$$

which can be expressed in the Green form:

$$
\frac{\Gamma(\boldsymbol{x})}{Y}=\int d^{2} y G_{L}(\boldsymbol{x}, \boldsymbol{y}) \rho(\boldsymbol{y})+U(\boldsymbol{x}),
$$

where $U(\boldsymbol{x})$ is an harmonic function on $\mathbb{P}$ that enforces the Neumann boundary conditions (10b). The calculation of the Green's function $G_{L}(\boldsymbol{x}, \boldsymbol{y})$ satisfying equation (12) can be reduced to the more familiar planar problem by conformally mapping the paraboloid $\mathbb{P}$ onto the unit disk of the complex plane (see Appendix A for a detailed explanation):

$$
G_{L}(\boldsymbol{x}, \boldsymbol{y})=\frac{1}{2 \pi} \log \left|\frac{z(\boldsymbol{x})-z(\boldsymbol{y})}{1-z(\boldsymbol{x}) \overline{z(\boldsymbol{y})}}\right|
$$

where $z(\boldsymbol{x})=\varrho e^{i \phi}$, a point in the unit disk of the complex plane, is the image of a point on the paraboloid under the conformal mapping. The conformal distance $\varrho$ is related to $r$ by:

$$
\varrho(r)=\lambda \frac{r e^{\sqrt{1+\kappa^{2} r^{2}}}}{1+\sqrt{1+\kappa^{2} r^{2}}},
$$

with $\lambda$ a scale factor which ensures that $\varrho(R)=1$. As explained in detail in Appendix A, the Green's function $G_{L}(\boldsymbol{x}, \boldsymbol{y})$, and hence the entire elastic free energy, depends only on the coefficients of the first fundamental form of the surface (i.e. the metric tensor $g_{i j}$ ). Thus the elastic energy associated with the defect interactions and thus the crystalline order is an intrinsic property of the manifold and so is invariant under local isometries. This observation, which might appear obvious in the case of isometric surfaces such the Euclidean plane and the cylinder, is quite remarkable when applied to more sophisticated isometric manifolds such as the catenoid and the helicoid or Scherk surfaces. We will take this point up again in Sec. IV.

Inserting Eq. (15) into Eq. (14), the function $\Gamma(\boldsymbol{x})$ can be expressed in the simple form:

$$
\frac{\Gamma(\boldsymbol{x})}{Y}=\frac{\pi}{3} \sum_{i=1}^{N} q_{i} G_{L}\left(\boldsymbol{x}, \boldsymbol{x}_{i}\right)-\Gamma_{s}(|\boldsymbol{x}|)+U(\boldsymbol{x})
$$

where the first term represents the bare contribution of the defects to the energy density and the second corresponds to the screening effect of the Gaussian curvature. Explicitly:

$$
\Gamma_{s}(|\boldsymbol{x}|)=\log \left(\frac{\alpha e^{\sqrt{1+\kappa^{2} r^{2}}}}{1+\sqrt{1+\kappa^{2} r^{2}}}\right)
$$

where $r=|\boldsymbol{x}|$ and

$$
\alpha=\frac{1+\sqrt{1+\kappa^{2} R^{2}}}{\exp \left(\sqrt{1+\kappa^{2} R^{2}}\right)}
$$

is a normalization constant depending on boundary radius $R$ and the ratio $\kappa$.

Fig. 1 shows a plot of the screening function $\Gamma_{s}(r)$ for different values of $\kappa$ in the range $1-2$. As expected the contribution due to Gaussian curvature is maximum at the origin of the paraboloid and drops to zero at the boundary.

The calculation of the harmonic function $U(\boldsymbol{x})$ requires a little more effort. If the crystal was defect-free (or populated by a perfectly isotropic distribution of defects) the function $U(\boldsymbol{x})$ would be azimuthally symmetric and constant on the boundary. By the maximum principle of harmonic functions, $U(\boldsymbol{x})$ would be then constant on the whole manifold and dependent only on $\kappa$ and the radius $R: U(\boldsymbol{x})=U_{\kappa, R}$. Such a constant can be determined by integrating $\Delta \chi(\boldsymbol{x})=\Gamma(\boldsymbol{x})$ and imposing the boundary

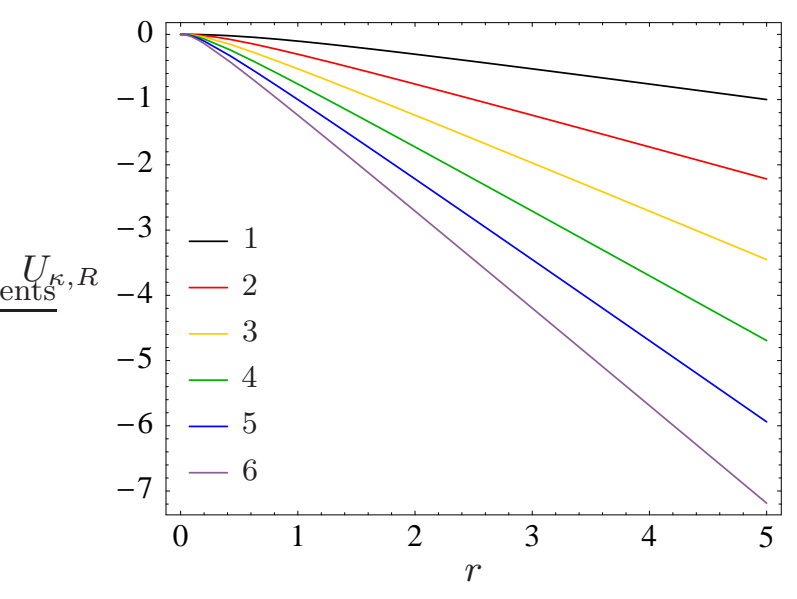

FIG. 2: The quantity $U_{\kappa, R}$ as a function of $R$ for different values of $\kappa$ in the range 1-6. 
condition (10b) [9]. This gives:

$$
U_{\kappa, R}=\frac{2 \pi}{A} \int_{0}^{R} d r \sqrt{g} \Gamma_{s}(r),
$$

where $A$ is the area of the paraboloid:

$$
A=\frac{2 \pi}{3 \kappa^{2}}\left[\left(1+\kappa^{2} R^{2}\right)^{\frac{3}{2}}-1\right] .
$$

As shown in Figure 2, the value of $U_{\kappa, R}$ quickly approaches the linear regime as the size of the radius increases:

$$
U_{\kappa, R} \approx-\frac{1}{4} \kappa R+\frac{1}{3}
$$

Then for a defect-free configuration, the contribution of the boundary to the energy density is a constant offset that persist even for large radii. In the presence of disclinations, on the other hand, the function $\chi(\boldsymbol{x})$ is no longer expected to be azimuthally symmetric and the harmonic function $U(\boldsymbol{x})$ will not be constant throughout the paraboloid. Introducing the harmonic kernel $H(\boldsymbol{x}, \boldsymbol{y})$ such that:

$$
U(\boldsymbol{x})=-\int d^{2} y H(\boldsymbol{x}, \boldsymbol{y}) \rho(\boldsymbol{y})
$$

the determination of $U(\boldsymbol{x})$ requires the calculation of the function $H(\boldsymbol{x}, \boldsymbol{y})$ associated with the Green's function of the weighted biharmonic operator arising from the conformal map of the paraboloid onto the unit disk of the complex plane. This problem has been intensively studied in the past few years by the mathematics community because it is connected with the extension of the maximum principle for the weighted biharmonic operator of the form $\Delta w^{-1} \Delta$ (see Hedenmalm et al. [15] for a good survey on this topic). In the case of radial weights, as arises from the conformal mapping of any surface of revolution, the function $H(\boldsymbol{x}, \boldsymbol{y})$ can be calculated explicitly (see Shimorin [16]). For the sake of completeness we report an exact expression of the harmonic kernel $H(\boldsymbol{x}, \boldsymbol{y})$ in Appendix B. The physical understanding of the solution (17), however, doesn't require the complete solution. As shown from the numerical data of Sec. III, the distribution of the defects along the boundary is predominately symmetric, and thus we expect the constant factor (23) to be the leading contribution from the boundary even in the general case.

For the ground state energy (zero temperature limit) that interests us we must minimize the energy (6) with respect to both the positions and the total number of defects. Since the energy density (17) depends on the difference between a curvature term and the defect density we expect the defects to arrange themselves so to approximately match the Gaussian curvature. A complete screening of the Gaussian curvature would yield a crystal with zero elastic energy at zero temperature. On the other hand the core energy associated with a generic number $N$ of disclinations will be linear in $N$ and so it
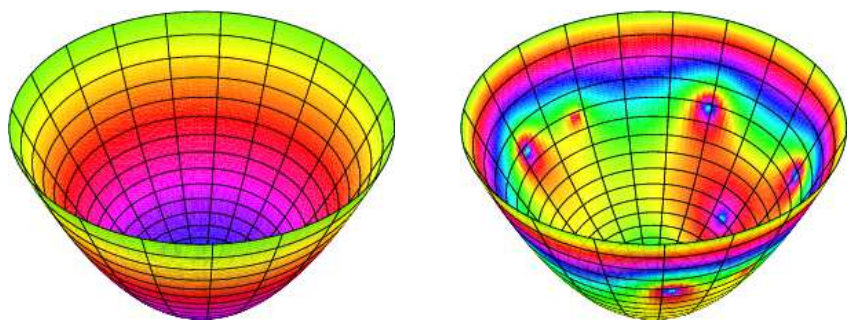

FIG. 3: Energy density $\Gamma^{2}(\boldsymbol{x})$ for two different defect distributions. The energy density corresponding to an isolated +1 -disclination at the origin is shown on the left. The defect distribution used for the figure on the right has been obtained from the numerical minimization of the Coulomb energy of a system of $V=50$ charges (see Sec. III).

will favor the fewest defects possible. The structure of the crystal at zero temperature will be governed therefore by the competition between the energy cost of creating a defect and the compensating screening effect of the Gaussian curvature. Once the distribution of the defects is known the elastic energy can be easily calculated by integrating the (18) on the whole paraboloid. In Fig. 3 we show an example of the energy density $\Gamma^{2}(\boldsymbol{x})$ corresponding to two different arrangements of defects on a paraboloid with $(\kappa=1.6$ and $R=1)$.

\section{B. Large Core Energies: Pyramidal Lattices}

In the regime of large core energies $F_{c} \gg F_{e l}$, the creation of defects is strongly penalized and the lattice necessarily has the minimum number of disclinations allowed by the topology of the paraboloidal substrate. From symmetry considerations we might expect the optimal distribution of defects to consist of $b+1$-disclinations arranged along the boundary at the base vertices of a $b$-gonal pyramid and a $b$-fold apex (of topological charge $\left.q_{0}=6-b\right)$ at the origin. The homogeneous boundary conditions adopted require the first term in Eq. (17) to vanish when $\boldsymbol{x}_{i} \in \partial \mathbb{P}$. In the minimal energy configuration then, the system has the freedom to tune the total number of defects along the boundary to minimize the elastic energy Eq. (8) for any given value of the ratio $\kappa$. This behavior is exclusive to manifolds with boundary and doesn't have any counterpart in crystals on compact surfaces like the sphere and the torus. In the following we will see how this minimization leads to properties which we believe to hold, in the most general sense, on any surface with boundary.

We will label a pyramidal configuration by $Y_{b}$, where $b$ denotes the number of base +1 -disclinations. The coordinates $(r, \phi)$ of the vertices are given by:

$$
Y_{b}: \quad\left\{(0, \text { any }),\left(R, \frac{2 \pi k}{b}\right)_{1 \leq k \leq b}\right\} .
$$

Using the Euler theorem one can show that it is possible 


\begin{tabular}{c|cccc}
\hline \hline$n$ & $Y_{3}$ & $Y_{4}$ & $Y_{5}$ & $Y_{6}$ \\
\hline 1 & 4 & 5 & 6 & 7 \\
2 & 10 & 13 & 16 & 19 \\
3 & 19 & 25 & 31 & 37 \\
4 & 31 & 41 & 51 & 61 \\
5 & 46 & 61 & 76 & 91 \\
6 & 64 & 85 & 106 & 127 \\
7 & 85 & 113 & 141 & 169 \\
8 & 109 & 145 & 181 & 217 \\
9 & 136 & 181 & 226 & 271 \\
10 & 166 & 221 & 276 & 331 \\
\hline \hline
\end{tabular}

TABLE I: Number of vertices $V$ for four different $Y_{b, n}$ families in the range $b \in[3,6]$ (tetrahedron, square, pentagonal and hexagonal pyramid) and $n \in[1,10]$.

to construct infinite families of polyhedra with the symmetry group $C_{b v}$ from the pyramidal backbone $Y_{b}$. The number of vertices is given by:

$$
V=\frac{1}{2} b n(n+1)+1,
$$

where $n$ is a positive integer which represents the number of edges (not necessarily of the same length) of the polyhedron which separates two neighboring disclinations. In the following we will refer to these polyhedra with the symbol $Y_{b, n}$. Fig. 4 illustrates two $Y_{b, n}$ lattices for the cases $b=4$ and $n=7$ (with $V=113$ ), and $b=5$ and $n=10(V=276)$. In Table I we report the number of vertices for the four simplest $Y_{b, n}$ polyhedra for $n \in[1,10]$. By a numerical minimization of the energy Eq.(8) one can establish that the $Y_{b}$ are indeed equilibrium configurations for $b \in[3,5]$, for some range of the parameters $\kappa$ and $R$. The cases of $b=5,6$ are particularly significant because they are characterized by an equal number of defects $(N=6)$ of the same topological charge $(q=1)$. The two configurations will be associated therefore with the same core energy $F_{c}$ and this introduces the possibility of a structural transition between $Y_{5}$ and $Y_{6}$ governed by the curvature ratio $\kappa$ and the boundary radius $R$. For fixed $R$ and small values of $\kappa$ the 6 -fold symmetric configuration $Y_{6}$ is the global minimum of the free energy Eq.(6). For $\kappa$ larger than some critical value $\kappa_{c}(R)$, however, the $Y_{6}$ crystal becomes unstable with respect to the 5 -fold symmetric configuration $Y_{5}$. A numerical calculation of the intersection point between the elastic energies of $Y_{5}$ and $Y_{6}$ for different values of $\kappa$ and $R$ allow us to construct the phase diagram shown in Fig. 5. The word "phase" in this context refers to the symmetry of the ground state configuration as a function of the geometrical system parameters $\kappa$ and $R$.

The scenario depicted in Fig. 5 can be understood heuristically by imagining a system of spherically symmetric equally sized subunits initially arranged on the surface of a planar disk $(\kappa=0)$. The most efficient packing of this system is clearly the one in which the subunits are arranged in a triangular lattice with six 3 -fold sites on the boundary at the vertices of a hexagon. If now we
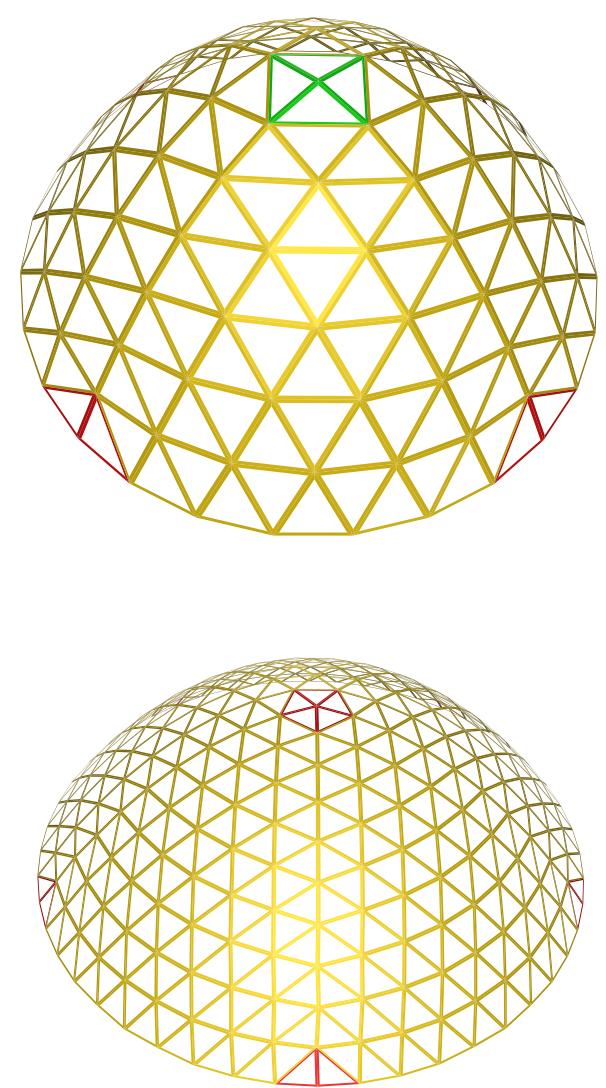

FIG. 4: Two examples of $Y_{b, n}$ triangulations of the paraboloid ( $Y_{4,7}$ on the top and $Y_{5,10}$ on the bottom). Plaquettes with disclinations are highlighted in red, for +1 -disclinations, and green for +2 -disclinations.

slightly deform the disk into a low-curvature paraboloid $(\kappa>0)$ we might expect the hexagonal configuration to persist for small values of $\kappa$. When the deformation is more pronounced, however, the curvature at the origin will be enough to support the existence of a 5 -fold vertex and the system will undergo a structural transition from the $Y_{6}$ to the $Y_{5}$ phase. In principle, if we keep increasing the curvature we might expect the crystal to undergo a further transition to the $Y_{4}$ phase. In this case, however, the core energy will also increase by a factor $4 / 3$ and so this is not generally possible in the regime in which $F_{c} \gg F_{e l}$. For intermediate regimes (i.e. $F_{c} \sim F_{e l}$ ), $Y_{5} \rightarrow Y_{4}$ and $Y_{4} \rightarrow Y_{3}$ transitions are also possible. The critical value of the parameters $\kappa$ and $R$, however, is not universal and will depend in detail on the values of the core energy and the Young modulus. 


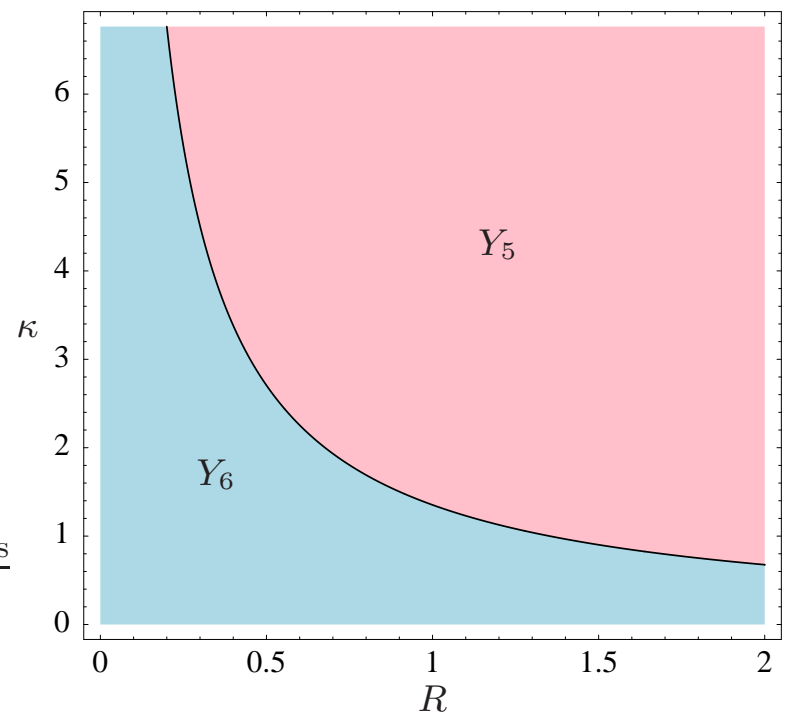

FIG. 5: Phase diagram in the large core energy regime. For small $\kappa$ the lattice preserves the 6 -fold rotational symmetry of the flat case. As the curvature at the origin increases the system undergoes a transition to the $Y_{5}$ phase.

\section{Small Core Energies: Scars And Coexistence}

When the core energy $F_{c}$ is small, the elastic energy Eq.(6) can be lowered by creating additional defects. In this section we present a simple argument for the approximate phase diagram of a paraboloidal crystal in this regime. A more detailed analysis of the minimal energy configurations following from the solution Eq.(17) will be reported elsewhere.

Let us assume that a fivefold disclination is sitting at the point $\boldsymbol{x}_{0}=\left(r_{0}, \phi_{0}\right)$ of $\mathbb{P}$. We can introduce a notion of distance on the paraboloid by setting up a system of geodesic polar coordinates $(s, \varphi)$ with origin at $\boldsymbol{x}_{0}$. We expect that the stress introduced by the defect is controlled by an effective disclination charge inside a circular domain $C_{L}$ of geodesic radius $L$ :

$$
q_{e f f}=q-\int_{0}^{2 \pi} d \varphi \int_{0}^{L} d s \sqrt{g} K(s, \varphi)
$$

where $q=\pi / 3$ is the charge of the isolated defect and the integral measures the screening due to the total Gaussian curvature within the domain. The metric tensor and the Gaussian curvature of a generic Riemannian manifold can be expressed in geodesic polar coordinates in the form (see for example Do Carmo [17]):

$$
\begin{gathered}
g_{i j}=\left(\begin{array}{ll}
1 & 0 \\
0 & G
\end{array}\right), \\
K(s, \varphi)=-\frac{\partial_{s}^{2} \sqrt{G}}{\sqrt{G}},
\end{gathered}
$$

where $G=\boldsymbol{g}_{\varphi} \cdot \boldsymbol{g}_{\varphi}$. Furthermore, an expansion of the metric around the origin $(0, \varphi)$ yields:

$$
\sqrt{G}=s-\frac{1}{6} K_{0} s^{3}+o\left(s^{5}\right) .
$$

For small distance from the origin, Eq.(26) becomes:

$$
\begin{aligned}
q_{e f f} & =q+\int_{0}^{2 \pi} d \varphi \int_{0}^{L} d s \partial_{s}^{2} \sqrt{G} \\
& =q-\pi K_{0} L^{2}+o\left(L^{4}\right) .
\end{aligned}
$$

The right hand side of Eq.(29) is a very general expression for the effective disclination charge at small distance and doesn't depend on the embedding manifold. If a grain boundary is radiating form the original disclination, we expect the spacing between consecutive dislocations to scale like $1 / q_{\text {eff }}$, with $a$ the lattice spacing [2]. When $q_{\text {eff }} \rightarrow 0^{+}$the dislocation spacing diverges and the grain boundary terminates. Since the Gaussian curvature is not constant on $\mathbb{P}$, the choice of the origin (i.e. the position of the central disclination along the grain boundary) affects the evaluation of $q_{e f f}$. One can identify upper and lower bounds by observing that:

$$
\begin{gathered}
\max _{r} K(r)=K(0)=\kappa^{2}, \\
\min _{r} K(r)=K(R)=\frac{\kappa^{2}}{\left(1+\kappa^{2} R^{2}\right)^{2}} .
\end{gathered}
$$

Unlike the case of surfaces with constant Gaussian curvature, we expect the phase diagram for paraboloidal crystals to consist of three regions separated by the curves:

$$
K_{0} L^{2}=\frac{1}{3} \quad K_{0}=K_{\min }, K_{\max } .
$$

When $L-L\left(K_{\min }\right) \rightarrow 0^{+}$the effective disclination charge goes to zero and the distance between two consecutive dislocations diverges at any point on $\mathbb{P}$. On the other hand if $L-L\left(K_{\max }\right) \rightarrow 0^{-}$, the disclination charge will prefer to be delocalized in the form of grain boundary scars. For $L\left(K_{\max }\right)<L<L\left(K_{\min }\right)$ the paraboloid will be equipped with both regions where the Gaussian curvature is high enough to support the existence of isolated disclinations and regions where the screening due to the curvature is no longer sufficient and the proliferation of grain boundary scars is energetically favored. This leads to a three region phase diagram in which the regime of isolated disclinations is separated from the delocalized regime of scars by a novel phase in which both isolated disclinations and scars coexist in different parts of the paraboloid according to the magnitude of the Gaussian curvature.

To compare this result with the structure of paraboloidal crystals obtained by numerical minimization of the Coulomb energy (see Sec. III) we need to measure the distance $L_{c}$ in terms of the lattice spacing $a$ and rephrase Eq.(31) as a condition on $a$ (or equivalently on the number of vertices $V$ ). To do this we note that in order for the domain $C_{L}$ to completely screen 


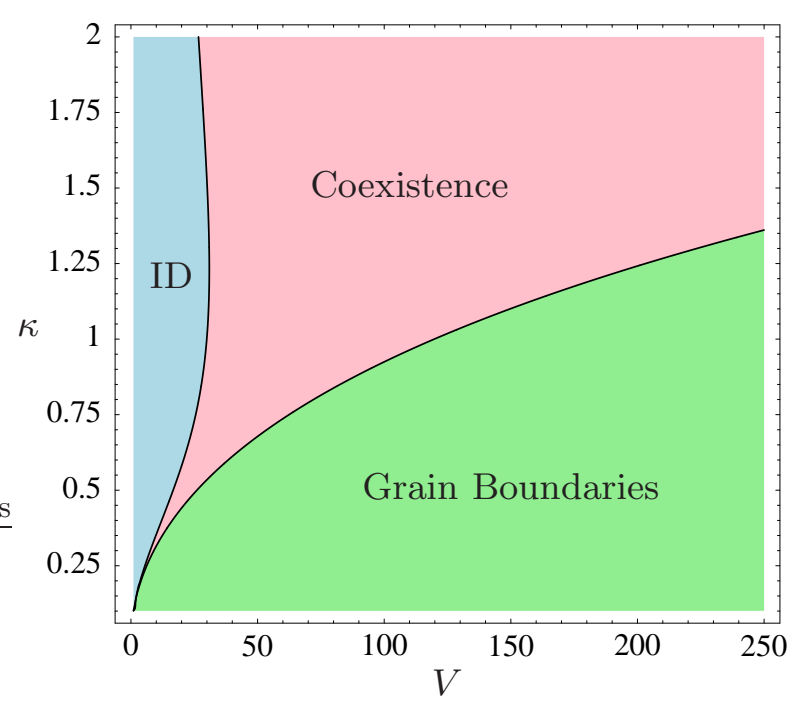

FIG. 6: Defect phase diagram for a paraboloidal crystal of radius $R=1$. The two critical lines that separate the isolated disclinations (ID) regime from the coexistence regime and the coexistence regime from the scar phase correspond to the solutions of Eq.(32) for $K_{0}=K_{\min }$ and $K_{0}=K_{\max }$, respectively.

the topological charge of the shortest scar possible (i.e. $5-7-5$ ), the geodesic radius $L$ has to be large enough to enclose the entire length of the scar. Calling $\ell$ the geodesic distance associated with a single lattice spacing $a$, we will then approximate $L \sim 3 \ell$. In the spirit of the local expansion Eq.(29), we can then approximate the neighborhood of the central point $\boldsymbol{x}_{0}$ with a spherical cap of radius $1 / \sqrt{K_{0}}$. With this choice we obtain:

$$
a^{2} \approx \frac{2}{K_{0}}\left[1-\cos \left(\ell \sqrt{K_{0}}\right)\right],
$$

which, combined with Eq.(31), gives an equation in $a$ and $\kappa$ for fixed $R$ :

$$
a^{2} \approx \frac{2}{K_{0}}\left(1-\cos \frac{1}{3 \sqrt{3}}\right) .
$$

The lattice spacing $a$ can be approximately expressed as a function of the number of vertices of the crystal by dividing the area $A$ of the paraboloid by the area of a hexagonal Voronoi cell of radius $a / 2$ :

$$
a^{2} \approx \frac{A}{\frac{\sqrt{3}}{2} V}
$$

The phase diagram arising from the solution of Eq.(32), together with the condition Eq.(34), is sketched in Fig. 6 for the case $R=1$. The two critical lines that separate the isolated defects (ID in the plot) regime from the coexistence regime and this one form the grain boundaries phase correspond respectively to the solutions for $K_{0}=K_{\min }$ and $K_{0}=K_{\max }$. The simplicity of the criteria used to derive Eqs.(32) and (34) doesn't allow us to predict the regions surrounding the critical lines with high numerical accuracy, but does provide a semiquantitative picture of the novel phenomenology of defects in non-Euclidean crystals that is generally supported by the numerical results presented in Sec. III.

\section{NUMERICAL EXPERIMENTS}

\section{A. Energy Minima And Complexity}

In the following section we report the results of a numerical minimization of a system of $V$ classical particles interacting via a Coulomb potential $E=\sum_{i<j} 1 /\left|\boldsymbol{x}_{i}-\boldsymbol{x}_{j}\right|$ on the surface of a paraboloid. The equilibrium configuration arising from this optimization problem can be viewed as a direct realization of a paraboloidal crystal and thus provides a testing ground for our analytical results.

The determination of the equilibrium properties of complex systems is complicated by the rich topography of the energy landscape, with its many, often deep, local minima (valleys) separated by high barriers (passes). The number of local minima grows rapidly with system size, making it increasingly difficult, or impossible, to find the global minimum.

The effort in solving a given global optimization problem is described by computational complexity theory. Locating the global minimum for a potential energy surface belongs to the class of problems known as NP-hard, for which there is no known algorithm that is certain to solve the problem within a time that scales as a power of the system size.

The Thomson problem [18-25] of finding the optimal configuration of $V$ interacting charges on a 2-sphere represents, in this context, a celebrated example of a hard optimization problem. The existence of novel arrays of topological defects in minimal energy configurations provides further insight into the structure of the energy landscape. Computer experiments on the Thomson problem indicate that, in the range $70 \leq V \leq 112$, the number of local minima for each value of $V$ grows exponentially: $\mathcal{N} \simeq 0.382 \exp (0.0497 V)$ [21]. This trend is believed to continue for larger values of $V$, making the determination of the global minimum a formidable computational challenge. In the case of the paraboloid we believe the prefactor in this scaling law is larger due to the additional constraint of the boundary.

\section{B. Paraboloidal Coulomb Crystals}

To construct equilibrium lattice configurations we adopted a parallel implementation of Differential Evolution (DE) [26] (see App. C for an introductory description of the algorithm together with the parallelization 


\begin{tabular}{c|ccccc}
\hline \hline$V$ & $V_{-2}$ & $V_{-1}$ & $V_{0}$ & $V_{1}$ & Energy \\
\hline 10 & 0 & 0 & 4 & 6 & 36.94485696974016 \\
20 & 0 & 4 & 6 & 10 & 179.5291483377297 \\
30 & 0 & 6 & 12 & 12 & 439.0497473530407 \\
40 & 2 & 5 & 18 & 15 & 818.8300625504069 \\
50 & 4 & 4 & 24 & 18 & 1321.878894548272 \\
60 & 2 & 10 & 28 & 20 & 1949.230291403783 \\
70 & 2 & 16 & 26 & 26 & 2701.959660541221 \\
80 & 3 & 17 & 31 & 29 & 3581.110585181344 \\
90 & 2 & 16 & 46 & 26 & 4588.364706108566 \\
100 & 3 & 15 & 55 & 27 & 5722.503370970009 \\
150 & 1 & 30 & 81 & 38 & 13323.70617345018 \\
200 & 3 & 35 & 115 & 47 & 24173.215803305491 \\
\hline \hline
\end{tabular}

TABLE II: Numerical data for twelve selected lattices. The quantities $V_{q}$ represent the number of vertices in the crystal with topological charge $q$.

strategy). The initial pool of candidate solutions is generated at the beginning of the simulation by randomly creating $N P=20 \mathrm{~V}$ configurations uniformly distributed over the whole search space $\{r \in[0, R]\} \otimes\{\phi \in[0,2 \pi]\}$. The population is then evolved by $3 \cdot 10^{5} \mathrm{DE}$ iterations on ten processors working in parallel.

In Fig. 7 we show the Voronoi lattice and the Delaunay triangulations for five selected systems up to $V=200$ particles. The lowest energy seen, together with the number of $n$-fold vertices, for each one of these lattices is reported in Table III A. In all the systems observed disclinations always appear clustered in either grain boundary scars or dislocations with the exception of isolated +1 -disclinations which appearing in the bulk as expected from the curvature screening argument discussed in Sec. II. The complex aggregation of defects along the boundary together with the presence of negatively charged clusters indicates that the effect of the boundary, in the case of relatively small systems like the ones simulated, is more drastic than predicted by the homogeneous boundary conditions Eq.(10a) and Eq.(10b). Even in the computationally expensive case of $V=100$, the distance between the origin and the boundary of the paraboloid is only four lattice spacings. In this situation we expect the distribution of particles along the boundary to play a major role in driving the order in the bulk.

For larger systems, such as $V=200$ (top of Fig. 7), the behavior of the particles in the bulk is less affected by the boundary and the crystalline order reflects more closely the free-boundary problem discussed in Sec. II. A comparison of the lattices in the first two rows of Fig. 7 , in particular, reveals substantial agreement with the scenario described in Sec. II B. For $\kappa=0.8$ and $V=200$, the defects are all localized along the boundary with the exception of one length-3 scar in the bulk at distance $r \approx 0.63$ from the center. For $\kappa=1.6$, the pattern of defects in the bulk is characterized by the coexistence of
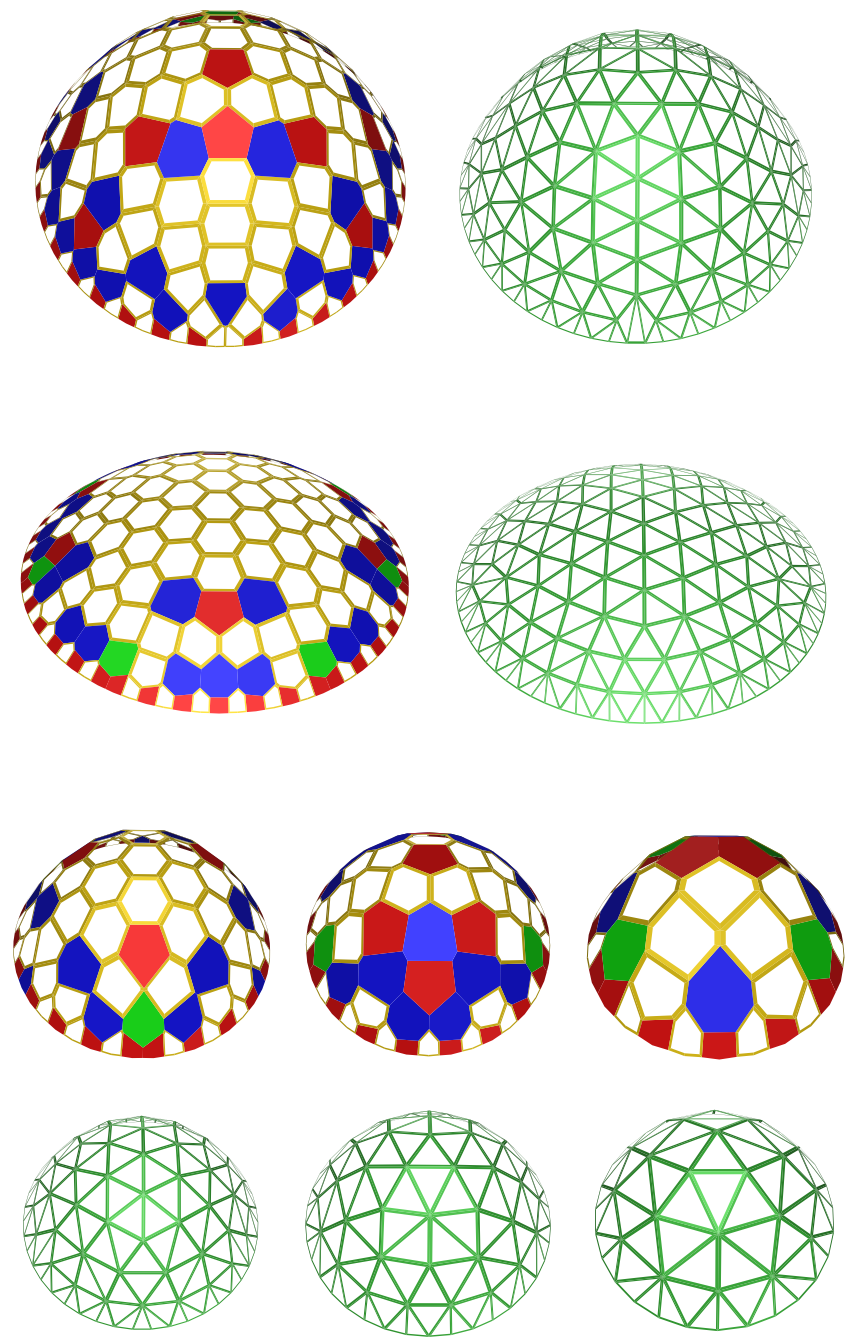

FIG. 7: Voronoi lattices and Delaunay triangulations for five selected systems from numerical simulations with $R=1$. The first row corresponds to $V=200$ and $\kappa=1.6$, while the second row is for $V=200$ and $\kappa=0.8$ (see Sec. IIIB for a discussion). In the bottom two rows $V=100,80,50$ with $\kappa=1.6$.

an isolated +1 -disclination at the origin and a length$5 W$-shaped scar displaced along a parallel one lattice spacing away from the central disclination. Apart from the evident difficulty in comparing the structures of small systems with those predicted from continuum elasticity theory, this behavior is consistent with the simple picture sketched in the phase-diagram of Fig. 6. The local 5 -fold symmetry at the origin of the $\kappa=1.6$ configuration, compared with 6 -fold symmetry for $\kappa=0.8$, suggests, as in the case of spherical crystals [27], that the complicated structure of defect clusters appearing in large systems is the result of the instability of the simpler $Y_{b, n}$ configurations from which they partially inherit their overall symmetry. A more accurate numerical verification of our theory remains a challenge for the future. 
The symmetry of the configurations presented in Fig. 7 deserves special attention. As for any surface of revolution, the circular paraboloid belongs possesses the symmetry group $O(2)$ of all rotations about a fixed point and reflections in any axis through that fixed point. Any given triangulation of the paraboloid may destroy the full rotational symmetry completely or just partially, leaving the system in one of the following two subgroups: the pyramidal group $C_{n v}$ or the reflection symmetry group $C_{s}$. In general we found the latter symmetry group for system sizes up to $V=200$ particles. The symmetry for larger system sizes is under investigation.

\section{CONCLUSIONS AND DISCUSSIONS}

We have analyzed both analytically and numerically the structure of a two-dimensional paraboloidal crystal as a specific realization of the class of crystalline structures on two-dimensional Riemannian manifolds with variable Gaussian curvature and boundary. Using the geometrical approach developed in [2] we found that the presence of variable Gaussian curvature, combined with the presence of a boundary, gives rise to a rich variety of phenomena which we believe to be generic. The freedom of tuning the optimal number of disclinations to place on the boundary, where the presence of defects doesn't cost additional energy, allows the crystal to undergo a structural transition controlled by the value of the maximum Gaussian curvature of the surface, in the regime where the creation of defects is energetically disfavored and disclinations are isolated in the crystal and far apart from each other. When the core energy of the defects is much smaller than the elastic energy and disclinations are allowed to proliferate, the presence of a variable Gaussian curvature is responsible for the existence of an intermediate phase in which both isolated defects and grain boundary scars coexist in the crystal in different regions according to the magnitude of the curvature.

By conformally mapping the paraboloid into the unit disk of the complex plane, we showed furthermore that the elastic energy of the crystal only depends on the coefficients of the first fundamental form of the surface and thus is an intrinsic property of the manifold which is invariant for local isometries. This property, which is somehow already contained in the linearity of the elastic theory adopted, discloses a deep and fascinating feature of curved crystals by requiring isometric surfaces (as the catenoid and the helicoid or the Scherk surfaces) to support the same crystalline order. From the other hand this observation can be used to set a bound in the completeness of our theory and the accuracy of linear elasticity in describing the physics of defects in curved crystals. We hope more work will be devoted to the understanding of these phenomena in the future.

\section{Acknowledgments}

This work was supported by the NFS through Grant No. DMR-0219292 (ITR) and through founds provided by Syracuse University.

\section{APPENDIX A: EVALUATION OF THE FUNCTIONS $G_{L}(\boldsymbol{x})$ AND $\Gamma(x)$}

In this appendix we discuss the calculation of $\Gamma(\boldsymbol{x})$ in Eq.(17). Consider the solution of the Green equation

$$
\Delta^{2} G_{2 L}(\boldsymbol{x}, \boldsymbol{y})=\delta(\boldsymbol{x}, \boldsymbol{y}) \quad \boldsymbol{x}, \boldsymbol{y} \in \mathbb{P}
$$

with homogeneous boundary conditions. In integral form this solution can be written:

$$
G_{2 L}(\boldsymbol{x}, \boldsymbol{y})=\int d^{2} z G_{L}(\boldsymbol{x}, \boldsymbol{z})\left[G_{L}(\boldsymbol{z}, \boldsymbol{y})-H(\boldsymbol{z}, \boldsymbol{y})\right]
$$

where $G_{L}(\boldsymbol{x}, \boldsymbol{y})$ is the solution of the Green-Dirichlet problem Eq.(12) and $H(\boldsymbol{x}, \boldsymbol{y})$ is the reproducing kernel of Eq.(23). As noted in Sec. II A, one can map the paraboloid $\mathbb{P}$ onto the unit disk of the complex plane and then employ the appropriate planar techniques (i.e. image charges). In general any simply connected twodimensional Riemannian manifold with a $C^{\infty}$-smooth metric $d s^{2}$ can be equipped with a set of local isothermal (or conformal) coordinates $(x, y)$ such that the metric is represented in the form $d s^{2}=w(x, y)\left(d x^{2}+d y^{2}\right)$ for some positive conformal weight $w$. In two dimensions this local system of isothermal coordinates can serve as a conformal chart for the unit disk $\mathbb{D}$ on the complex plane. Calling $z=\varrho e^{i \phi}$ the new metric will be

$$
d s^{2}=w(z)\left(d \varrho^{2}+\varrho^{2} d \phi^{2}\right) .
$$

The conformal factor $w(z)$ can be found by equating the metric (A3) with the original one. At this point it is worth treating the problem in a slightly more general form. Consider the case of a generic surface of revolution of the form:

$$
\left\{\begin{array}{l}
x=\xi(r) \cos \phi \\
y=\xi(r) \cos \phi \\
z=\eta(r)
\end{array}\right.
$$

with $r \in[0, \infty[$ and $\phi \in[0,2 \pi]$. The metric of the surface (A4) will be:

$$
d s^{2}=E d r^{2}+2 F d r d \phi+G d \phi^{2}
$$

where

$$
\left\{\begin{array}{l}
E=\xi^{\prime 2}+\eta^{2} \\
F=0 \\
G=\xi^{2}
\end{array}\right.
$$


are the coefficients of the first fundamental form of the surface (A4). Equating (A5) and (A3) one finds immediately:

$$
w(\varrho)=\left[\frac{\xi(r)}{\varrho}\right]^{2},
$$

with $\varrho$ and $r$ related by the differential equation:

$$
\frac{d \varrho}{d r} \pm \sqrt{\frac{E}{G}} \varrho=0,
$$

whose solution is given by:

$$
\varrho=\exp \left(\mp \int d r \sqrt{E / G}\right) .
$$

The sign of the exponent and the integration constant in (A9), can be tuned to obtain the desired scale and direction of the conformal map. It is easy to show that, in the new coordinates, the Laplace operator takes the form:

$$
\Delta=w^{-1} \Delta_{z},
$$

in which $\Delta_{z}$ is the Laplacian in the Euclidean metric:

$$
\gamma=\left(\begin{array}{cc}
1 & 0 \\
0 & \varrho^{2}
\end{array}\right) \text {. }
$$

With this new set up, the Green equation (A1) can be easily written in the form:

$$
\Delta_{z} w^{-1} \Delta_{z} G_{2 L}(z, \zeta)=\delta(z, \zeta),
$$

where $\zeta=r^{\prime} e^{i \phi^{\prime}}$ is a second generic point of the complex plane and $\delta(z, \zeta)$ has the same meaning as in Sec. II A with respect the Euclidean metric $\gamma$. The differential operator $\Delta w^{-1} \Delta$ is known in analysis as weighted biharmonic operator. Analogously, the Green-Dirichlet problem (12) becomes:

$$
\left\{\begin{array}{ll}
\Delta G_{L}(z, \cdot)=\delta(z, \cdot) & z \in \mathbb{D} \\
G_{L}(z, \cdot)=0 & z \in \partial \mathbb{D}
\end{array},\right.
$$

so that:

$$
G_{L}(z, \zeta)=\frac{1}{2 \pi} \log \left|\frac{z-\zeta}{1-z \bar{\zeta}}\right| .
$$

It must be stressed that so far we didn't explicitly use the geometry of the paraboloid. What has been said, therefore, holds for any surface of revolution which can be conformally mapped onto the unit disk. Furthermore, as anticipated in Sec. II A, the conformal distance $\varrho$, which completely embodies the geometry of the surface, depends only on the coefficients $E$ and $G$ of the first fundamental form.

In the particular case of the paraboloid we have:

$$
\varrho=\lambda \frac{r e^{\sqrt{1+\kappa^{2} r^{2}}}}{1+\sqrt{1+\kappa^{2} r^{2}}},
$$

$$
\lambda=\frac{1+\sqrt{1+\kappa^{2} R^{2}}}{R \exp \left(\sqrt{1+\kappa^{2} R^{2}}\right)} .
$$

To obtain the expression for $\Gamma(\boldsymbol{x})$ given in Eq.(17) we are left with the task of calculating the integral:

$$
\Gamma_{s}(\boldsymbol{r})=\Gamma_{s, 1}(\boldsymbol{x})-\Gamma_{s, 2}(\boldsymbol{r}),
$$

where

$$
\begin{aligned}
& \Gamma_{s, 1}(\boldsymbol{x})=\frac{1}{2 \pi} \int d \phi^{\prime} d r^{\prime} \sqrt{g} K\left(r^{\prime}\right) \log |z-\zeta|, \\
& \Gamma_{s, 2}(\boldsymbol{x})=\frac{1}{2 \pi} \int d \phi^{\prime} d r^{\prime} \sqrt{g} K\left(r^{\prime}\right) \log |1-z \bar{\zeta}| .
\end{aligned}
$$

For this purpose we can use the expansion:

$$
\log |z-\zeta|=\log \varrho_{>}-\sum_{n=1}^{\infty} \frac{1}{n}\left(\frac{\varrho_{<}}{\varrho_{>}}\right)^{n} \cos n \delta \phi,
$$

where $\varrho_{>}\left(\varrho_{<}\right)$represents the largest (smallest) modulus between $z$ and $\zeta$, while $\delta \phi=\phi-\phi^{\prime}$. The factorization of the angular variables in Eq.(A18), together with the pure radial dependence of the Gaussian curvature and $\sqrt{g}$, makes the angular dependence of $\Gamma_{s, 1}$ vanish, so that we have:

$$
\begin{aligned}
\Gamma_{s, 1}(r) & =\log \varrho(r) \int_{0}^{r} d r^{\prime} \frac{\kappa^{2} r^{\prime}}{\left(1+\kappa^{2} r^{\prime 2}\right)^{\frac{3}{2}}} \\
& +\int_{r}^{R} d r^{\prime} \frac{\kappa^{2} r^{\prime}}{\left(1+\kappa^{2} r^{\prime 2}\right)^{\frac{3}{2}}} \log \varrho\left(r^{\prime}\right),
\end{aligned}
$$

which integrated by parts gives:

$$
\Gamma_{s, 1}(r)=\log \left(\frac{\alpha e^{\sqrt{1+\kappa^{2} r^{2}}}}{1+\sqrt{1+\kappa^{2} r^{2}}}\right) .
$$

Using an expansion similar to (A18) it is also possible to prove that

$$
\log |1-z \bar{\zeta}|=-\sum_{n=1}^{\infty} \frac{1}{n}\left(\varrho \varrho^{\prime}\right)^{n} \cos \delta \phi,
$$

which integrated over the surface of the paraboloid gives $\Gamma_{s, 2}=0$. This last conclusion, combined with Eq. (A20), yields Eq.(18).

\section{APPENDIX B: THE HARMONIC KERNEL}

In this appendix we give the exact expression for the harmonic kernel appearing in Eq.(23). This expression has been found by Shimorin in the more general case of the calculation of the Green function for the weighted biharmonic operator with radial weight $w(z)=w\left(|z|^{2}\right)$ [16]. As in the previous appendix we call $z=\rho(r) e^{i \phi}$ and $\zeta=\rho^{\prime}\left(r^{\prime}\right) e^{i \phi^{\prime}}$ two points of the complex plane that are images of the points $(r, \phi)$ and $\left(r^{\prime}, \phi^{\prime}\right)$ of $\mathbb{P}$ under 

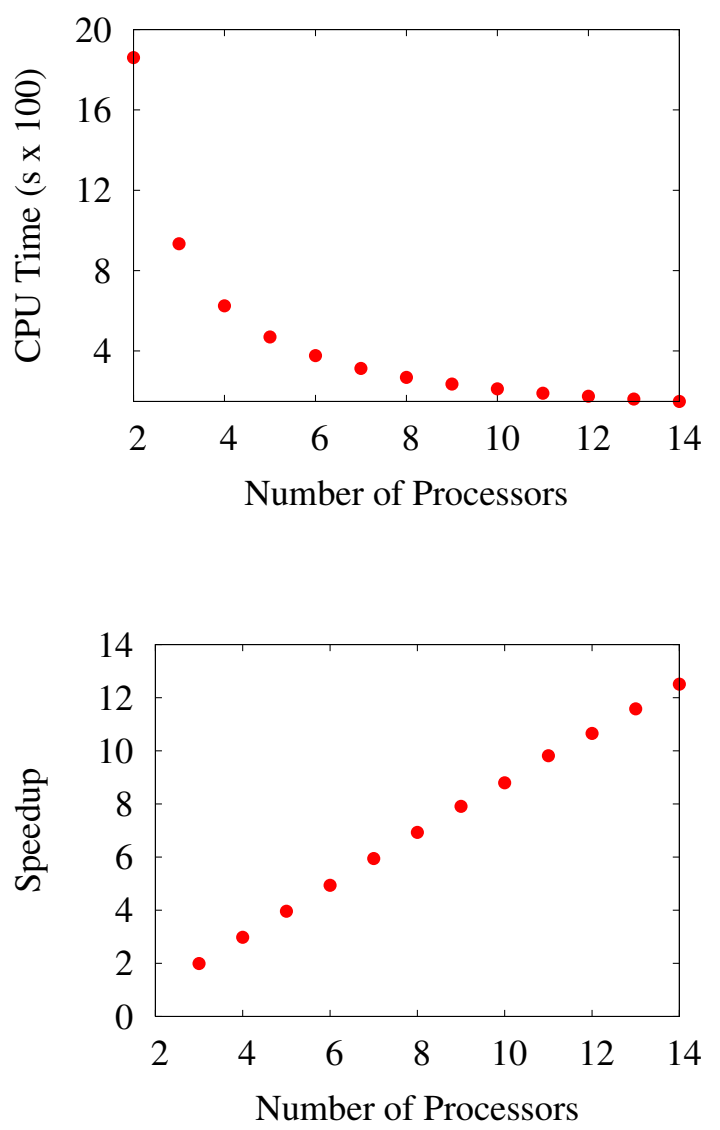

FIG. 8: CPU-Time and speedup (i.e. the time employed by $n$ processors to accomplished a given number of iterations divided by the time employed by a single machine to achieve the same task).

the conformal transformation (16). The harmonic kernel $H(z, \zeta)$ can be written in integral form as:

$$
H(z, \zeta)=-\int_{|\zeta|}^{1} \frac{d t}{\pi t} \int_{0}^{t} d s \sqrt{g} k\left(\frac{\varrho^{2}(s)}{t^{2}} \zeta \bar{z}\right)
$$

in which:

$$
k(z \bar{\zeta})=\sum_{n \geq 0} \frac{(z \bar{\zeta})^{n}}{c_{n}}+\sum_{n<0} \frac{(\overline{z \zeta})^{|n|}}{c_{|n|}}
$$

where the coefficients $c_{n}$ are given by:

$$
c_{n}=2 \int_{0}^{1} d s \sqrt{g} \varrho^{2 n}(s) .
$$

\section{APPENDIX C: OPTIMIZATION VIA PARALLEL DIFFERENTIAL EVOLUTION}

Many of the techniques proposed to determine the crystalline structure of systems of interacting particles, as in the Thomson problem, are based on local optimization procedures such as steepest descent, conjugate gradient and the quasi-Newton method. Such methods belong to the class of line-search algorithms for multidimensional non-linear programming problems. They can be described, in general, as a sequence of line minimizations of an objective function along a set of directions that are generated differently in different algorithms. Besides the well known local convergence properties of these methods, they are generally unable to locate the global minimum since they inherently approach the closest local minimum for a given set of initial conditions.

To avoid the misconvergence problem described we adopt the Differential Evolution (DE) algorithm of Storn and Price [28]. This algorithm, which has been successfully applied to several optimization problems in engineering [29], belongs to the family of evolutionary algorithms which are considerably faster than other stochastic optimization methods, such as simulated annealing and genetic algorithms, and more likely to find the correct global minimum. These methods heuristically mimic biological evolution by implementing natural selection and the principle of "survival of the fittest". An adaptive search procedure based on a population of candidate solutions is used. Iterations involve a competitive selection that drops the poorer solutions. The remaining pool of candidates are perturbed (or mutated) in order to generate trial individuals and then recombined with other solutions by a swap of the components. The recombination and mutation moves are applied sequentially; their aim is to generate new solutions that are biased towards subsets of the search space in which good, although not necessarily globally optimized, solutions have already been found.

An essential feature of Differential Evolution is the establishment of genetic diversity, which helps to maximize the probability of finding the true global minimum and to avoid misconvergence. One begins with a large population of individuals uniformly distributed in the search space. A good choice, in practice, is to choose the number of individuals to be an order of magnitude more than the number of variables in the problem. The price one pays is a dramatic slowing down of the algorithm when applied to large scale optimization. Considerable effort has therefore been made in the past ten years to develop parallel implementations of evolutionary algorithms aimed at reducing the overall time to completion of the task by distributing the work on different processors working in parallel. More recently some researchers have conjectured that some parallelizations of a task improve the quality of the solution obtained for a given overall amount of work (e.g. emergent computation).

The Island Model is a popular choice among parallelization strategies and is implemented within a message passing model. It consists of dividing the initial population into several sub-populations and letting each of them evolve independently on a single machine for a predetermined number of iterations (called the epoch). The exchange of genetic information is promoted by swapping 
individuals between different sub-populations at the end of each epoch. In the present work the migration strategy consists in swapping the best individual of each subpopulation with a randomly selected individual on an- other island with the ring topology chosen for the connectivity between islands. This choice allowed us to achieve a substantial reduction of the CPU time and a linear speedup (see Fig. 8).
[1] D. R. Nelson and L. Peliti, J. Physique 48, 1085 (1987).

[2] M. J. Bowick, D. R. Nelson, and A. Travesset, Phys. Rev. B 62, 8738 (2000), cond-mat/9911379.

[3] M. J. Bowick, A. Cacciuto, D. R. Nelson, and A. Travesset, Phys. Rev. Lett. 89, 185502 (2002), condmat/0206144.

[4] A. R. Bausch, M. J. Bowick, A. Cacciuto, A. D. Dinsmore, M. F. Hsu, D. R. Nelson, M. G. Nikolaides, A. Travesset, and D. A. Weitz, Science 299, 1716 (2003), cond-mat/0303289.

[5] A. D. Dinsmore, M. F. Hsu, M. G. Nikolaides, M. Marquez, A. R. Bausch, and D. A. Weitz, Science 298, 1006 (2002).

[6] A. Travesset, Phys. Rev. E 72, 03611 (2005), condmat/0601586.

[7] Y. Chushak and A. Travesset, Europhys. Lett. 72, 767 (2005), cond-mat/0603777.

[8] M. J. Bowick, A. Cacciuto, D. R. Nelson, and A. Travesset, Phys. Rev. B 73, 024115 (2006), cond-mat/0509777.

[9] V. Vitelli, J. B. Lucks, and D. R. Nelson, Proc. Natl. Acad. Sci. USA 103, 12323 (2006), cond-mat/0604203.

[10] J. Lidmar, L. Mirny, and D. R. Nelson, Phys. Rev. E 68, 051910 (2003), cond-mat/0306741.

[11] T. T. Nguyen, R. F. Bruinsma, and W. M. Gelbart (2005), physics/0506127.

[12] E. J. Kramer, Nature 437, 824 (2005).

[13] M. J. Bowick and A. Travesset, J. Phys. A 34, 1535 (2001), cond-mat/0005356.

[14] M. J. Bowick, Statistical Mechanics of Membranes and Surfaces (World Scientific, Singapore, 2004), 2nd ed.

[15] H. Hedenmalm, S. Jakobsson, and S. Shimorin, C. R.
Acad. Sci. Paris Sér. I Math. 328, 973 (1999), URL http: //www.esi.ac.at/preprints/ESI-Preprints.html.

[16] S. Shimorin, J. Math. Sci. 92, 4404 (1998).

[17] M. D. Carmo, Differential geometry of curves and surfaces (Prentice-Hall, 1976).

[18] J. J. Thomson, Philos. Mag. 7, 237 (1904).

[19] E. B. Saff and A. B. J. Kuijlaars, Math. Intelligencer 19, 5 (1997).

[20] D. P. Hardin and E. B. Saff, Notices of the Am. Math. Soc. 51, 1186 (2005).

[21] T. Erber and M. G. Hockney, Phys. Rev. Lett. 74, 1482 (1995).

[22] J. R. Morris, D. M. Deaven, and K. M. Ho, Phys. Rev. B 54, R1740 (1996).

[23] E. L. Altschuler, T. J. Williams, E. R. Ratner, R. Tipton, R. Stong, F. Dowla, and F. Woote, Phys. Rev. Lett. 78, 2681 (1997).

[24] E. L. Altschuler and A. P. Garrido, Phys. Rev. E 71, 047703 (2005), cond-mat/0408355.

[25] D. J. Wales and S. Ulker, Phys. Rev. B 74, 212101 (2006).

[26] Our optimization software is written in C and MPI. The code is available on-line, URL http://physics.syr. edu/ lgiomi/paraboloid/phess-v1.1.tgz.

[27] T. Einert, P. Lipowsky, J. Schilling, M. J. Bowick, and A. Bausch, Langmuir 21, 12076 (2005), condmat/0506741.

[28] R. Storn and K. Price, J. Glob. Opt. 11, 341 (1997).

[29] G. C. Onwubolu and B. Babu, New Optimization Techniques in Engineering (Studies in Fuzziness and Soft Computing) (Springer, 2004). 